\title{
COVID-19 vaccine-induced Radiation Recall Phenomenon: report of a case
}

Mozhdeh Sepaskhah ${ }^{1}$, Fatemeh Ansari $\mathrm{Asl}^{2}$, Mehrnoosh Taheri ${ }^{1}$, and Mojgan Akbarzadeh Jahromi $^{3}$

${ }^{1} 1$ - Department of Dermatology, School of Medicine, Shiraz University of Medical Sciences, Shiraz, Iran

21- Department of Dermatology, School of Medicine, Shiraz University of Medical Sciences, Shiraz, Iran.

${ }^{3} 3$ - Department of Pathology, School of Medicine, Shiraz University of Medical Sciences, Shiraz, Iran

December 7, 2021

\begin{abstract}
Radiation Recall Phenomenon (RRP) is an inflammatory process in the site of irradiation, induced by physical and medical agents. Few cases of RRP in the skin and lung have been reported after COVID-19 vaccination. Here, we report radiation recall dermatitis after both doses of inactivated SARS-CoV-2 vaccine (Sinopharm, China).
\end{abstract}

\section{Introduction}

Radiation Recall Phenomenon (RRP) is an inflammatory disorder triggered by some medications and physical agents at the site of previous irradiation. This phenomenon is reported in the skin and other organs, like lung, muscle, and intestine, and is mainly precipitated by anti-cancer medications; however, other drugs can also induce RRP (e.g., some antibiotics, statins, letrozole, tamoxifen). ${ }^{1,2}$

Recently a few cases of RRP have been reported after different types of COVID-19 vaccine in the skin and lung. ${ }^{3-5}$

Here, we report a case of radiation recall dermatitis after inactivated SARS-CoV-2 vaccine (Sinopharm, China), repeated after rechallenge in the second dose of vaccine.

\section{Case report}

A 50-year-old woman presented to our outpatient dermatology clinic with an erythematous, pruritic skin rash over the upper chest and neck one month before referral. The lesions started one week after the second dose of the SARS-CoV-2 vaccine (Sinopharm, China). Similar lesions (less extensive and less symptomatic) had developed after the first dose of vaccine, injected three weeks before the second dose.

The patient is a known case of breast cancer (invasive ductal carcinoma) of the left breast that had undergone radical mastectomy and post-operation chemotherapy and radiotherapy 15 years ago. Since then, she has had a routine follow up without any relapse of breast cancer.

On examination of the skin, relatively ill-defined erythematous plaque with focal scaling and mild lichenification was observed on the upper chest, on the irradiation window area marked by tattoo (Figure 1a). A skin punch biopsy was performed and the histopathological examination revealed hyperkeratosis, parakeratosis, 
mild irregular acanthosis, mild spongiosis, focal basal cell degeneration with few Civatte bodies, and high apoptotic cells. The upper dermis was infiltrated by perivascular lymphocytes and eosinophils and contained dilated blood vessels (Figure 2a-d). Some mast cells were also visible in the upper dermis (Figure 3).

According to the clinical and histopathological features, diagnosis of radiation recall dermatitis was made, and she was treated with oral prednisolone (15 mg daily) for three days, once-daily application of mometasone furoate ointment, and frequent application of emollient. The signs and symptoms improved in a 10-day follow-up. (Figure 1b)

The patient signed written informed consent to permit the publication of the case report without identifying data and to use the photography for publication. The researchers committed to maintaining the patient confidentiality. Institutional ethics committee approved the case report (ethics code: IR.sums.med.rec.1400.325).

\section{Discussion}

The radiation recall phenomenon represents an inflammatory process triggered by different agents in the previously irradiated body area. The most common triggering agents are drugs, especially anti-tumor ones. However, other medications have also been discussed as causes of RRP, including antibiotics ${ }^{1}$, $\operatorname{simvastatin}^{6}$, letrozole ${ }^{7}$, and tamoxifen ${ }^{8}$. The inflammation can involve not only the skin but also the lung, esophagus, small intestine, and some other organs. ${ }^{2}$

The radiation recall phenomenon had not been reported after any vaccination before the COVID-19 vaccination was started. Few reports of RRP after COVID-19 vaccination have been recently published. ${ }^{3-5}$ The radiation recall dermatitis occurred after the adenovirus vector vaccine (Oxford-AstraZeneca, UK) ${ }^{5}$, inactivated virus vaccine (Sinovac Coronavac, China) ${ }^{3}$, and RNA virus vaccine (Moderna, USA) ${ }^{4}$. Our case developed radiation recall dermatitis after injection of an inactivated virus vaccine (Sinopharm, China).

The previous cases of radiation recall dermatitis after COVID-19 vaccination presented with well-demarcated, erythematous, and indurated plaques ${ }^{3}$ and lesions with marked erythema and dry desquamation ${ }^{5}$.

Although only some of the previous cases of radiation recall dermatitis have undergone skin biopsy, some common features are described in the reported cases, including vacuolar changes in the dermo-epidermal junction, apoptotic epidermal cells, vascular dilatation, and dermal infiltration of lymphocytes and eosinophils. 2, 9 The same features were observed in the histopathological examination of our patient.

Ristic hypothesized the role of mast cells in the pathogenesis of RRD. ${ }^{10}$ Increased number of mast cells in the dermal inflammatory infiltration of our case may favor this hypothesis.

The time-lapse between radiation and RRP in our patient (15 years) was among the most prolonged periods presented in the literature ${ }^{2}$, but the time between the insult and development of the rash (7 days) was in concordance with most of previous cases. ${ }^{2}$ The radiation recall rash developed from three hours to five days after COVID-19 vaccine injection. ${ }^{3-5}$

The recurrence of RRP on re-exposure with the causative agent is controversial. ${ }^{1}$ The reported cases of RRP after COVID-19 vaccination developed the rash either only after the injection of the second dose of vaccine (and not the first dose) or after the first dose and before the injection of the second dose of vaccine ${ }^{3-5}$; so, the possibility of recurrence on rechallenge was absent or not assessable. Nevertheless, our patient experienced RRP after the injection of both vaccine doses, with a more severe reaction after the second dose.

The radiation recall phenomenon has been treated with oral and/or topical corticosteroids, antibiotics, emollients, and analgesics ${ }^{2}$, and the rash in our patient improved by a short course of oral and topical steroids.

\section{Conclusion}

There may be an increasing incidence of RRP after the COVID-19 vaccination. So, the physicians should be cautious about this diagnosis after the COVID-19 vaccine injection to recognize and treat it promptly. 


\section{Conflict of interest}

The authors declare no conflict of interest.

\section{Author Contributions}

Mozhdeh Sepaskhah: Clinical diagnosis of the case, treatment planning, writing the manuscript draft, final version approval

Fatemeh Ansari Asl: Data acquisition, patient follow-up, critical review of the draft, final version approval Mehrnoosh Taheri: Clinical diagnosis of the case, critical review of the draft, final version approval

Mojgan Akbarzadeh Jahromi: Pathological confirmation of the diagnosis, critical review of the draft, final version approval

\section{References}

1. Camidge R, Price A. Characterizing the phenomenon of radiation recall dermatitis. Radiother Oncol. 2001;59(3):237-45.

2. Azria D, Magné N, Zouhair A, Castadot P, Culine S, Ychou M, et al. Radiation recall: a well recognized but neglected phenomenon. Cancer Treat Rev. 2005;31(7):555-70.

3. Afacan E, Öğut B, Üstün P, Şentürk E, Yazıcı O, Adışen E. Radiation recall dermatitis triggered by inactivated COVID-19 vaccine. Clin Exp Dermatol. 2021.

4. Steber CR, Ponnatapura J, Hughes RT, Farris MK. Rapid Development of Clinically Symptomatic Radiation Recall Pneumonitis Immediately Following COVID-19 Vaccination. Cureus. 2021;13(4):e14303.

5. Stewart R, McDowell L. Radiation Recall Phenomenon Following COVID-19 Vaccination. Int J Radiat Oncol Biol Phys. 2021.

6. Abadir R, Liebmann J. Radiation reaction recall following simvastatin therapy: a new observation. Clin Oncol. 1995;7(5):325-6.

7. Foster LM, Mahoney ME, Harmon MW, Allen JW, Luh JY. Radiation recall reaction with letrozole therapy in breast cancer. Clin Breast Cancer. 2014;14(3):e95-7.

8. Rhee J, Kim GE, Lee CH, Kwon JM, Han SH, Kim YS, et al. Radiation recall dermatitis induced by tamoxifen during adjuvant breast cancer treatment. Radiat Oncol J. 2014;32(4):262-5.

9. Stieb S, Riesterer O, Brüssow C, Pestalozzi B, Guckenberger M, Weiler S. Radiation recall dermatitis induced by sorafenib : A case study and review of the literature. Strahlenther Onkol. 2016;192(5):342-8.

10. Ristić B. Radiation recall dermatitis. Int J Dermatol. 2004;43(9):627-31.

\section{Figure Legends}

Figure 1. Clinical features. a. Erythematous, ill-defined plaques on the upper chest and neck (previous radiotherapy site), with focal dry desquamation b. Significant improvement of the lesions after 10 days of treatment

Figure 2. Histopathological features. a. Hyperkeratosis, focal spongiosis, and upper dermal perivascular infiltration [Hematoxylin and eosin $(\mathrm{H} \& \mathrm{E})$ stain, $40 \times$ ] b. Hyperkeratosis, parakeratosis, focal spongiosis (black arrow), focal basal cell degeneration (green arrow), and dilated vessels in the dermis (blue arrows) (H\&E stain, 100×) c. Basal cell degeneration (green arrow), and high apoptotic cells (black arrow) in the epidermis (H\&E stain, 400×) d. Superficial dermal perivascular, mononuclear cell infiltrate admixed with eosinophils (green arrows) and dilated blood vessels (black arrow) (H\&E stain, 400 $\times$ )

Figure 3. Histopathological features. Increased mast cell infiltration (red arrows) in the upper dermal infiltration (Giemsa stain, $400 \times$ ) 

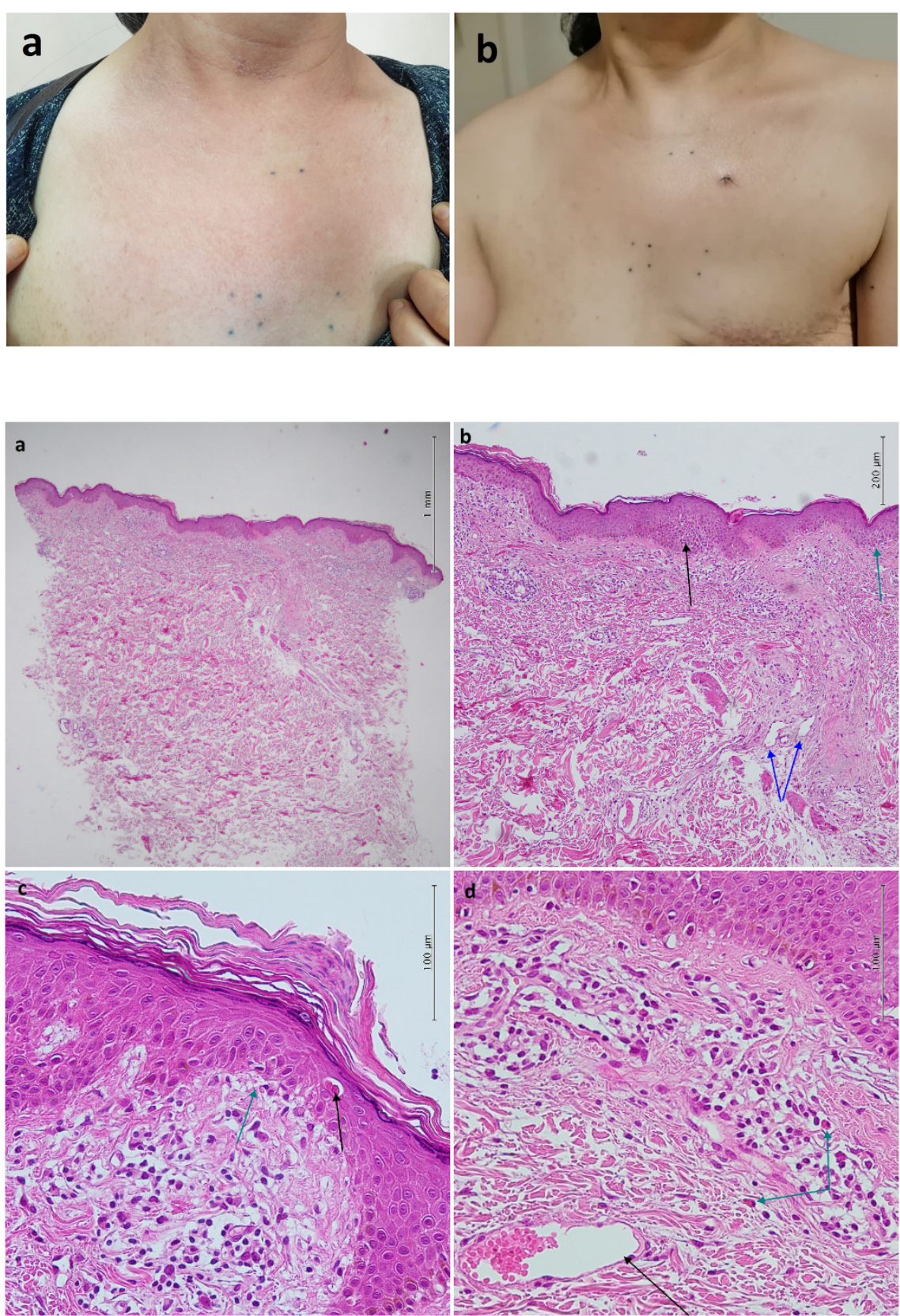


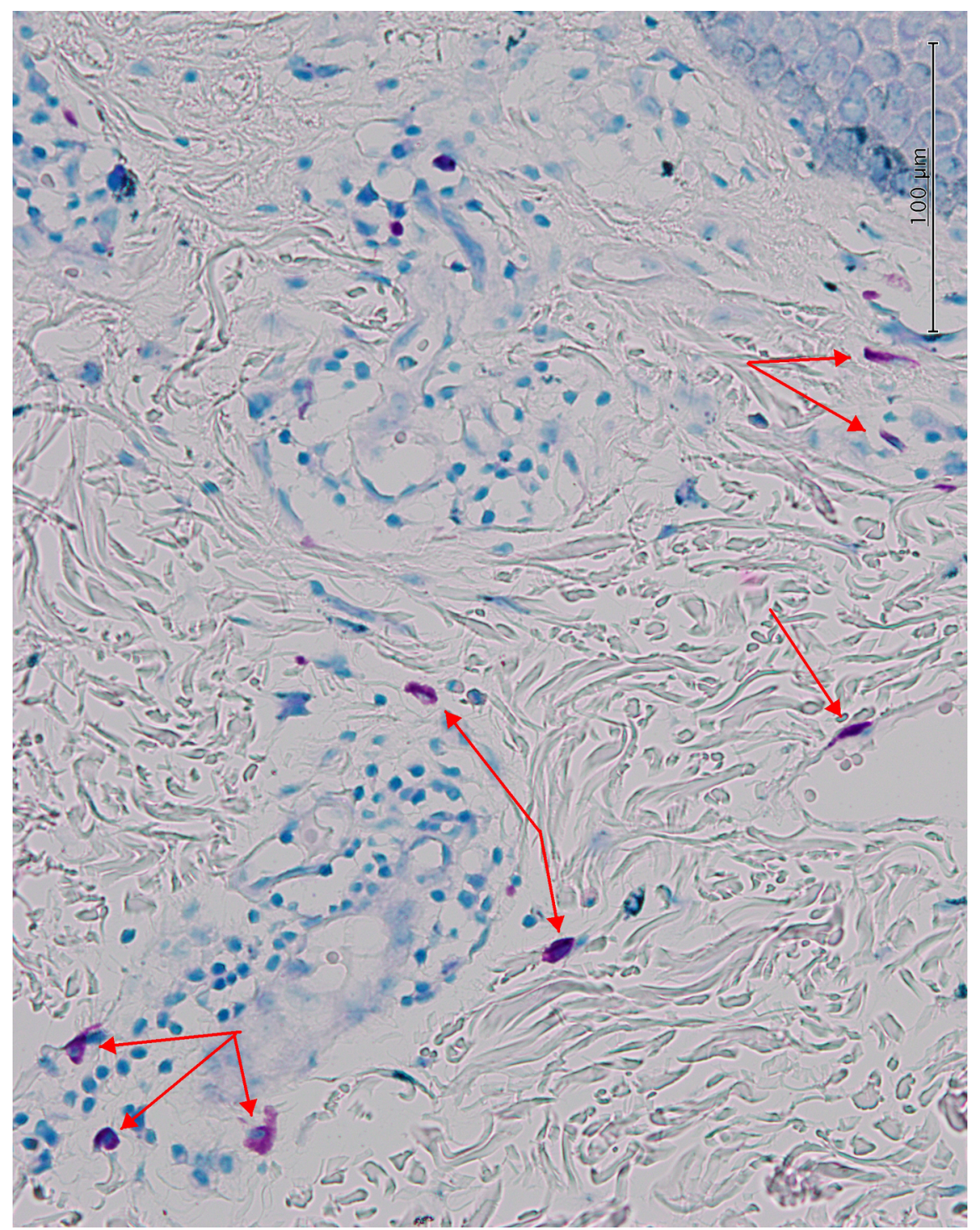

